\title{
Performance Tuning for Optimal Backup Process on Database Server
}

\author{
Yosua Dwi Raharjo ${ }^{1}$, Abba Suganda Girsang ${ }^{2}$ \\ ${ }^{1}$ Computer Science Department, BINUS Graduate Program - Master of Computer Science, Bina \\ Nusantara University, Jakarta, Indonesia 11480,Email : yosua.raharjo@binus.ac.id \\ ${ }^{1}$ Computer Science Department, BINUS Graduate Program - Master of Computer Science, Bina \\ Nusantara University, Jakarta, Indonesia 11480,Email : agirsang@ binus.edu
}

\begin{abstract}
With the current rise of the new technology and the need of data maintenance over the looming risk of hardware failures or external threats, this paper aims to reassess the most optimal backup process on IT Services by experimenting of different parameter tuning. The aim is to suggest which combination is deemed suitable for a certain business requirement based on a real study case. There are various studies and research on comparing the performance of how one configuration could affect the total performance with a certain metrics they want to measure. But currently there are no works of study of assessing how does each parameter tuning would affect the backup performance on Oracle database, since it is only categorized on high-level language (basic, high, low, medium).

This study would use the data and requirement from one of the largest government-based company in Indonesia using Oracle backup tools: RMAN
\end{abstract}

Key words: Backup, Database, Oracle, RMAN.

\section{INTRODUCTION}

Data is currently considered one of the most important assets for any organizations in nowadays. Data is so important for running and improving the business systems, to the point of people saying that data has changed the company workflow [1], all in order to attain and retain the data. Hence data maintaining is now considered crucial as one of the key points in IT services.

In order to protect the Availability and Integrity of the data on 'High' level, many measures [2], various in the practices, are done to prevent such as theft, modification, writing error or any risk inducing activity which may cause any loss for the company. These measures should be designed to not only protect the database from external harm, but also to prepare the restoration function should something happen to the data. This is why periodical backup is a very important procedure that must be done in any database. Backup is a process of creating a duplicate instance by copying the data in a new archive, so that the data won't be lost should the primary database crashes, corrupted or lost [3].

There are two main purposes of a database backup. First of course is for the data restoration as stated above, the second is to access information from a certain point on the past that may have been changed or updated on the current primary database. These purposes would be taking almost the same amount of size, if not more, of what the primary database currently is. This pushed the research and studies on how to design or help backup procedures, especially with companies with such large amount of data; such with data de-duplication and compression, so they can achieve this target.

In order to execute high availability and optimal disaster recovery strategy, a help from a trustworthy and reliable backup system has a very important role to fill. For organizations which use Oracle based database, Oracle has provided a comprehensive backup and recovery manager named RMAN [4]. RMAN gives the user a certain degree of freedom of tuning to help adjusting the user need. This study is aimed using a study case of a real company, to find the best suited tuned parameter of backup which can be suggested to company for its backup procedure due to the importance of assessing of how to choose a technology [5], that can help organization to suppress cost, size, resource and also the recovery time.

\section{RELATED WORKS}

\subsection{Theoritical Background}

\section{A. Business Overview}

The Case study of this experiment would be taken on one of the largest government-based company in Indonesia. The requirement of the backup is performed daily and there are major transactions on the system every day with no service time limit per day.

The company regulates that backups are to be performed with following schedule; Full back up once a week, and 
incremental backup once a day while retaining the previous day's backup for up to 7 days before the weekly full backup as a measure for the data loss should the system fails.

According to [6], there are two kinds of backups; hot and cold depending on the database status. Backup which done on an inactive database to ensure the consistency of the data when being restored in called 'Cold Backup' and in opposite, when done on an active running database so that the service could still run on the database are named 'Hot Backup'.

Due to the activity of the database of this company is running 24 hours, this experiment would be performed on a Hot backup condition [7].

For such, there are currently two database backup tools in the Oracle which can help meet the company needs, such with OS and RMAN. While OS and RMAN provides the same result, what made it differs is the database activity state. OS can only suitable for Cold Backup. Meanwhile RMAN is suitable for Hot Backup which is required for this case.

\section{B. Recovery Manager (RMAN)}

RMAN is a default provided tool which developed by the Oracle to backup the database which are needed for recovery should the primary database fails [8]. It covers the following types of files:

a. Data file, and image copy of datafile

b. Control file, and image copy of control file

c. Archived redo logs

d. Server parameters file

e. Backup pieces, which is automatically generated by the RMAN

It is also noteworthy that compression feature on RMAN can decrease the size of the database file up to $50-60 \%$ of its original file.

There are a few different kinds of backup procedure type which are available by the RMAN:

\section{Full Backup}

This procedure would backup the whole process, data, and any other configuration which exists in the primary database. Depending on the primary size, the setback of this type of procedure is the time and resource, as well as the need of a large size of storage.

\section{Archivelog Backup}

As long the database in in the ARCHIVELOG mode and backups (figure 1) the archive log along with the data files, inconsistent backups became the main reason for backup and restore [9].

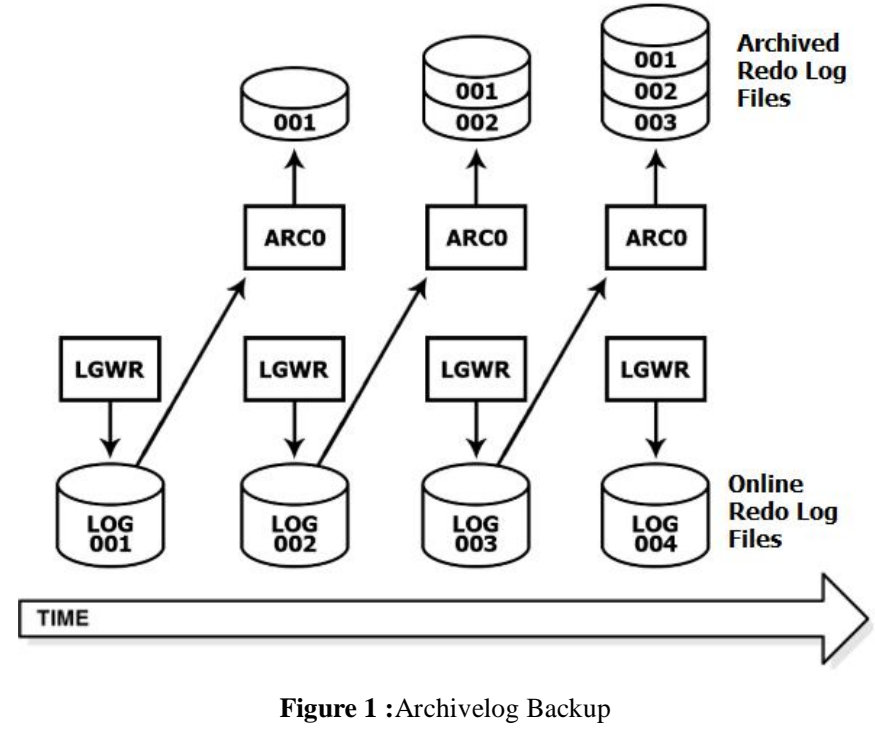

A consistent backup is the one of the most important strategy for backing up most of the database, these archive log backups are the incremental data which can serve for the recovery file.

\section{RPO and RTO}

The strategy for Disaster Recovery must be based on the Business Continuity Plan (BCP) which would state the target Recovery Point Objective (RPO) and the Recovery Time Objective (RTO)

RPO refers to how much of data can we restore, meanwhile RTO refers to how long we can restore the data to its original instance. These two objectives relate closely to how long would the backup takes in each process, and the results of the file size to be restored to achieve that time.

In order to achieve the state of "Zero Data Loss" when an emergency happens, an optimal backup process to achieve a faster with acceptable degree of compression is needed.

\section{RMAN Parameter Tuning}

There are a few parameters which need to be set by the user to optimize their backup process to adjust in order to meet the company needs.

\section{Compression Method}

RMAN would use a binary algorithm for compression process before writing the data into a set named backup set [10].

This compression result is similar to the vendor's compression results, though sadly there are official statements from the oracle regarding the compression ratio for each algorithm parameters. 
There are four types of binary compression settings on RMAN: Basic, Low, Medium and High. Here are the performance table1 of how the compression mode correlates with the backup size and the CPU usage [11].

\begin{tabular}{|l|l|l|}
\multicolumn{1}{c}{ Table 2: RMAN Binary Compression Matrix } \\
\begin{tabular}{|l|l|} 
RMAN \\
Compression \\
Type
\end{tabular} & $\begin{array}{c}\text { Compression } \\
\text { Algorithm Used }\end{array}$ & \multicolumn{1}{c|}{ Algorithm Description } \\
\hline BASIC & BZIP2 (LVL1) & $\begin{array}{l}\text { compression ratio in the range of } \\
\text { MEDIUM, but slower }\end{array}$ \\
\hline LOW & LZO & smallest compression ratio, fastest \\
\hline MEDIUM & ZLIB & $\begin{array}{l}\text { good compression ratio, slower } \\
\text { than LOW }\end{array}$ \\
\hline HIGH & BZIP2 (LVL9) & $\begin{array}{l}\text { highest compression ratio, } \\
\text { slowest }\end{array}$ \\
\hline
\end{tabular}

Bzip2 : bzip2 is a free and open-source file compression program that uses the Burrows-Wheeler algorithm [12].

LZO : a lossless data compression algorithm that is focused on decompression speed [13].

ZLIB : zlib only supports one algorithm, called DEFLATE, that is a variation of LZ77 (Lempel-Ziv 1977), this algorithm provides good compression on a wide variety of data with minimal use of system resources [14].

There is no official record for the aimed size and ratio of compression, but there is an experiment done with following result [15]:

Table 3: RMAN Compression Result Sample

\begin{tabular}{|c|c|c|}
\hline $\begin{array}{c}\text { Compression } \\
\text { Algorithm }\end{array}$ & $\begin{array}{c}\text { Size of } \\
\text { Compressed } \\
\text { Backup }\end{array}$ & Time Taken \\
\hline No & $\begin{array}{c}900 \mathrm{MB} \\
\text { (Uncompressed) }\end{array}$ & 2 Minutes \\
\hline BASIC & $280 \mathrm{MB}$ & 3.5 Minutes \\
\hline HIGH & $210 \mathrm{MB}$ & 7 Minutes \\
\hline MEDIUM & $240 \mathrm{MB}$ & 4.5 Minutes \\
\hline
\end{tabular}

\section{Multiplexing/Parallel Processing}

Multiplexing is a process to speed up the process of writing multiple data on a single disk, since there are limitations when we only backup a single client/sub clients. This process may not fully utilize the drive outputs or even the tape speed, hence multiplexing [4].

By default, the data multiplexed is set by the default of 8 MAXOPENFILES and 64 FILESPERSET.

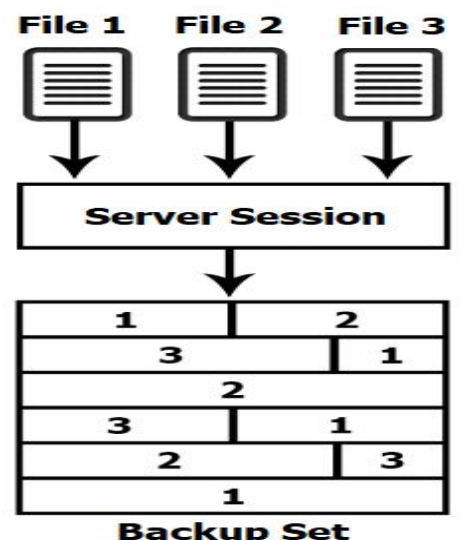

Figure 1: Multiplexing Schema

To illustrate it simple, hereby is the following schema with 3 Multiplexing condition (MAXOPENFILES $=3$ )

\section{Storage Disk Type}

There no one would deny a better hard drive would help a lot everything that requires a computational process. Hard drive types are especially important to select since it would then be the storage of keeping the most important data. Currently the most known hard disk types are SATA, SAS and SSD [16].

Hereby are the following key differences between SATA, SAS, dan SSD [17]:

a. SATA (Serial ATA)

From all the type of hard disk available, SATA types of hard disk are usually priced lower and could answer to the general needs of data storage on a PC.

The reason is because SATA are well known for their large capacity and a good power efficiency management for data storing compared to its later SAS.

This type of hard disk also has no qualms of keeping any kinds of data format such as emails, files, webs to archives. The following reasons make SATA so popular, especially for the storage system of a home PC

\section{b. SAS (Serial Attached SCSI)}

The winning point of SAS Hard drives are the transfer speed and the durability. A SAS is optimized for important application of organization so that it can run $24 / 7$ with the high speed transfer of 15.000 RPM, but they usually come in small storage capacity, making it not suitable for data storage in long term.

c. SSD (Solid State Drives)

SSD is a new hard disk type which offers $100 x$ throughput compared to its predecessor. 
Compatible with also any types of data, it is important to note that the main components of SSD are not made of spindle like SATA and SAS. This not only reduced the risk of disk failure and much less power consumed when writing the data, but also overcomes the speed (since there are no spinning) and durability.

The setback for SSD is that the price for one unit can be far above its two predecessor, making it less obtainable for any company scaled storage

\subsection{Previous Research}

There are various studies and research on comparing the performance of how one configuration could affect the total performance with a certain metrics they want to measure. Such as [3], performed a study on how SSD assist Backup and Recovery for Database, The Table 3 show the existing methods supported by varying layers with the result on each metrics.

Table 3 :Summary of existing methods for the database backup and recovery (FS: file system, DB: database)

\begin{tabular}{|c|c|c|c|c|}
\hline Methods & Mysqldump & Xtrabackup & BTRFS & LVM \\
\hline Type & logical & physical & physical & physical \\
\hline Layer & DB & DB & FS & block \\
\hline $\begin{array}{c}\text { Backup } \\
\text { speed }\end{array}$ & slow & slow & fast & fast \\
\hline $\begin{array}{c}\text { Restore } \\
\text { speed }\end{array}$ & slow & medium & fast & fast \\
\hline FS/OS & all & all & \multicolumn{2}{|c|}{$\begin{array}{c}\text { BTFRS/LVM } \\
\text { supported only }\end{array}$} \\
\hline
\end{tabular}

There is also a research on how oracle based on backup method mechanism [7], where the study focuses on how can oracle backup manager can do and elaborate the steps which is possible to take on each method (such as incremental or cumulative backup.

\begin{tabular}{|l|cccccc|c|c|}
\hline Changed \\
Blocks
\end{tabular}

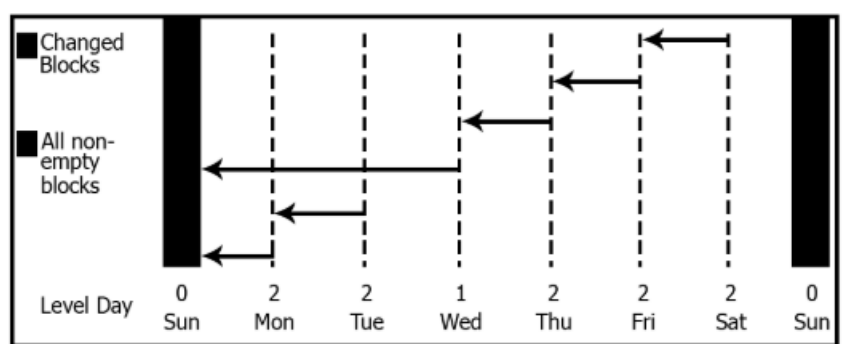

Figure 3: Cumulative vs Incremental Backup system on Oracle But currently there are no works of study of assessing how does each parameter tuning would affect the backup performance on Oracle database, since it is only categorized on high-level language (basic, high, low, medium), this study wants to assess on experiment level on how does the performance of each method using Oracle RMAN, and be able to suggest the best tuned result for the study case.

\section{METHODOLOGY}

With defining the correct tuning configuration for each of the parameter above, this study expects to be able to suggest which parameter combination with the best compatibility to answer the study case needs for "Zero Data Loss" in case the need arise.

The experiment for the backup database would be done on RMAN tools from Oracle, and it would be done on Hot Backup schema with Full Backup Scenario.

The parameters which would be taken to consideration are as follow: Storage Service Level, Parallel Process (Multiplexing) and Compression Level.

The database which is going to be experimented is totaling in 800GB size and restricted to RMDB types. Backup experiments will give results:

Table 4: Experiment Result Model

\begin{tabular}{|c|c|c|c|c|c|c|}
\hline $\begin{array}{c}\text { Compressio } \\
n\end{array}$ & Multiplexing & $\begin{array}{l}\text { Output } \\
\text { Size } \\
\text { (GB) }\end{array}$ & $\begin{array}{c}\text { Output } \\
\text { Size } \\
\text { (GB) }\end{array}$ & $\begin{array}{c}\text { Time } \\
\text { Elapsed } \\
\text { (GB) }\end{array}$ & $\begin{array}{l}\text { Output } \\
\text { Rate } \\
\text { (GB) }\end{array}$ & $\begin{array}{l}\text { CPU } \\
\text { Usage } \\
(\%)\end{array}$ \\
\hline \multirow{4}{*}{ BASIC } & $1 \mathrm{x}$ & $\mathrm{X}$ & $\mathrm{X}$ & $\mathrm{X}$ & $\mathrm{X}$ & $X$ \\
\hline & $8 x$ & $\mathrm{X}$ & $\mathrm{X}$ & $\mathrm{X}$ & $\mathrm{X}$ & $X$ \\
\hline & $16 \mathrm{x}$ & $\mathrm{X}$ & $\mathrm{X}$ & $\mathrm{X}$ & $\mathrm{X}$ & $\mathrm{X}$ \\
\hline & $24 x$ & $\mathrm{X}$ & $\mathrm{X}$ & $\mathrm{X}$ & $\mathrm{X}$ & $\mathrm{X}$ \\
\hline \multirow{4}{*}{ LOW } & $1 \mathrm{x}$ & $\mathrm{X}$ & $\mathrm{X}$ & $\mathrm{X}$ & $\mathrm{X}$ & $X$ \\
\hline & $8 \mathrm{x}$ & $\mathrm{X}$ & $\mathrm{X}$ & $\mathrm{X}$ & $\mathrm{X}$ & $\mathrm{X}$ \\
\hline & $16 x$ & $\mathrm{X}$ & $\mathrm{X}$ & $\mathrm{X}$ & $\mathrm{X}$ & $\mathrm{X}$ \\
\hline & $24 x$ & $\mathrm{X}$ & $\mathrm{X}$ & $\mathrm{X}$ & $\mathrm{X}$ & $X$ \\
\hline \multirow{4}{*}{ MEDIUM } & $1 \mathrm{x}$ & $\mathrm{X}$ & $\mathrm{X}$ & $\mathrm{X}$ & $\mathrm{X}$ & $\mathrm{X}$ \\
\hline & $8 x$ & $\mathrm{X}$ & $\mathrm{X}$ & $\mathrm{X}$ & $\mathrm{X}$ & $\mathrm{X}$ \\
\hline & $16 x$ & $\mathrm{X}$ & $\mathrm{X}$ & $\mathrm{X}$ & $\mathrm{X}$ & $\mathrm{X}$ \\
\hline & $24 x$ & $\mathrm{X}$ & $\mathrm{X}$ & $\mathrm{X}$ & $\mathrm{X}$ & $\mathrm{X}$ \\
\hline \multirow{4}{*}{$\mathrm{HIGH}$} & $1 \mathrm{x}$ & $\mathrm{X}$ & $\mathrm{X}$ & $\mathrm{X}$ & $\mathrm{X}$ & $\mathrm{X}$ \\
\hline & $8 x$ & $X$ & $\mathrm{X}$ & $\mathrm{X}$ & $\mathrm{X}$ & $X$ \\
\hline & $16 x$ & $\mathrm{X}$ & $\mathrm{X}$ & $\mathrm{X}$ & $\mathrm{X}$ & $\mathrm{X}$ \\
\hline & $24 x$ & $\mathrm{X}$ & $\mathrm{X}$ & $\mathrm{X}$ & $\mathrm{X}$ & $\mathrm{X}$ \\
\hline
\end{tabular}

Then it will be converted into scores based on threshold defined: 


\begin{tabular}{|c|c|c|c|c|c|c|}
\hline $\begin{array}{c}\text { Compressio } \\
\mathbf{n}\end{array}$ & $\begin{array}{c}\text { Multiplexin } \\
\mathbf{g}\end{array}$ & $\begin{array}{c}\text { Output } \\
\text { Size } \\
\text { (Score) }\end{array}$ & $\begin{array}{c}\text { Time } \\
\text { Elapsed } \\
\text { (Score) }\end{array}$ & $\begin{array}{c}\text { Output } \\
\text { Rate } \\
\text { (Score) }\end{array}$ & $\begin{array}{c}\text { CPU } \\
\text { Usage } \\
\text { (Score) }\end{array}$ & Total \\
\hline \multirow{4}{*}{ BASIC } & $1 \mathrm{x}$ & $\mathrm{X}$ & $\mathrm{X}$ & $\mathrm{X}$ & $\mathrm{X}$ & $\mathrm{X}$ \\
\cline { 2 - 7 } & $8 \mathrm{x}$ & $\mathrm{X}$ & $\mathrm{X}$ & $\mathrm{X}$ & $\mathrm{X}$ & $\mathrm{X}$ \\
\cline { 2 - 7 } & $16 \mathrm{x}$ & $\mathrm{X}$ & $\mathrm{X}$ & $\mathrm{X}$ & $\mathrm{X}$ & $\mathrm{X}$ \\
\cline { 2 - 7 } & $24 \mathrm{x}$ & $\mathrm{X}$ & $\mathrm{X}$ & $\mathrm{X}$ & $\mathrm{X}$ & $\mathrm{X}$ \\
\hline \multirow{4}{*}{ LOW } & $1 \mathrm{x}$ & $\mathrm{X}$ & $\mathrm{X}$ & $\mathrm{X}$ & $\mathrm{X}$ & $\mathrm{X}$ \\
\cline { 2 - 7 } & $8 \mathrm{x}$ & $\mathrm{X}$ & $\mathrm{X}$ & $\mathrm{X}$ & $\mathrm{X}$ & $\mathrm{X}$ \\
\cline { 2 - 7 } & $16 \mathrm{x}$ & $\mathrm{X}$ & $\mathrm{X}$ & $\mathrm{X}$ & $\mathrm{X}$ & $\mathrm{X}$ \\
\cline { 2 - 7 } & $24 \mathrm{x}$ & $\mathrm{X}$ & $\mathrm{X}$ & $\mathrm{X}$ & $\mathrm{X}$ & $\mathrm{X}$ \\
\hline \multirow{5}{*}{ MEDIUM } & $1 \mathrm{x}$ & $\mathrm{X}$ & $\mathrm{X}$ & $\mathrm{X}$ & $\mathrm{X}$ & $\mathrm{X}$ \\
\cline { 2 - 7 } & $8 \mathrm{x}$ & $\mathrm{X}$ & $\mathrm{X}$ & $\mathrm{X}$ & $\mathrm{X}$ & $\mathrm{X}$ \\
\cline { 2 - 7 } & $16 \mathrm{x}$ & $\mathrm{X}$ & $\mathrm{X}$ & $\mathrm{X}$ & $\mathrm{X}$ & $\mathrm{X}$ \\
\cline { 2 - 7 } & $24 \mathrm{x}$ & $\mathrm{X}$ & $\mathrm{X}$ & $\mathrm{X}$ & $\mathrm{X}$ & $\mathrm{X}$ \\
\hline \multirow{5}{*}{ HIGH } & $1 \mathrm{x}$ & $\mathrm{X}$ & $\mathrm{X}$ & $\mathrm{X}$ & $\mathrm{X}$ & $\mathrm{X}$ \\
\cline { 2 - 7 } & $8 \mathrm{x}$ & $\mathrm{X}$ & $\mathrm{X}$ & $\mathrm{X}$ & $\mathrm{X}$ & $\mathrm{X}$ \\
\cline { 2 - 7 } & $16 \mathrm{x}$ & $\mathrm{X}$ & $\mathrm{X}$ & $\mathrm{X}$ & $\mathrm{X}$ & $\mathrm{X}$ \\
\cline { 2 - 7 } & $24 \mathrm{x}$ & $\mathrm{X}$ & $\mathrm{X}$ & $\mathrm{X}$ & $\mathrm{X}$ & $\mathrm{X}$ \\
\hline
\end{tabular}

With the need of the company, we tried to assess the ideal result for scoring our results. The scoring would follow with each result assessment in this threshold:

Table 6: Scoring Threshold (Output Size and Output Rate)

\begin{tabular}{|c|c|c|c|c|}
\hline \multirow{2}{*}{ Score } & \multicolumn{2}{|c|}{ Output Size } & \multicolumn{2}{c|}{ Output Rate } \\
\cline { 2 - 5 } & Min & Max & Min & Max \\
\hline 1 & 700 & $>700$ & 0 & 1 \\
\hline 2 & 450 & 700 & 1 & 5 \\
\hline 3 & 200 & 450 & 5 & 10 \\
\hline 4 & 160 & 200 & 10 & 45 \\
\hline 5 & 140 & 160 & 45 & 90 \\
\hline 6 & 120 & 140 & 90 & 130 \\
\hline 7 & 100 & 120 & 130 & 150 \\
\hline 8 & 90 & 100 & 150 & 300 \\
\hline 9 & 80 & 90 & 300 & 450 \\
\hline 10 & 0 & 80 & 450 & $>450$ \\
\hline
\end{tabular}

Table 7: Scoring Threshold (Time Elapsed and CPU Usage)

\begin{tabular}{|c|c|c|c|c|}
\hline \multirow{2}{*}{ Score } & \multicolumn{2}{|c|}{ Time Elapsed } & \multicolumn{2}{c|}{ CPU Usage } \\
\cline { 2 - 5 } & Min & Max & Min & Max \\
\hline 1 & 600 & $>600$ & 70 & 100 \\
\hline 2 & 240 & 600 & 50 & 70 \\
\hline 3 & 180 & 240 & 40 & 50 \\
\hline 4 & 120 & 180 & 35 & 40 \\
\hline 5 & 60 & 120 & 30 & 35 \\
\hline 6 & 30 & 60 & 25 & 30 \\
\hline 7 & 18 & 30 & 20 & 25 \\
\hline 8 & 12 & 18 & 16 & 20 \\
\hline 9 & 6 & 12 & 12 & 16 \\
\hline 10 & 0 & 6 & 0 & 12 \\
\hline
\end{tabular}

Output size: the smaller output size value will give better scoring value. Output rate: the bigger output rate value will give better scoring value
Time elapsed: the faster backup process will give better scoring value

CPU Usage: the smaller CPU used for backup process will give better scoring value

\section{EXPERIMENT AND RESULT}

Hereby is the specification of the Database Server we are going to use in this study:

Database Server Specification:

Exadata X4-2 (6 node):

- CPU: Intel(R) Xeon(R) CPU E5-2697 v2 @ 2.70GHz 24 core

- Memory: 256 GB

- OS: Oracle Linux Server release 5.10

- RDBMS: Oracle Linux Enterprise 11.2.0.4

- Size database: $812,82 \mathrm{~GB}$

VMAX Storage Server Specification:

- CPU: Intel(R) Xeon(R) CPU E5-2603 v4 @ $1.70 \mathrm{GHz} 2$ core

- Memory: $16 \mathrm{~GB}$

- OS: Linux CentOS release 6.8 (Final)

- Capacity: 300TB

Monitoring Server Specification:

- CPU: Intel(R) Xeon(R) CPU E5-2690 0 @ 2.90GHz 16 core

- Memory: 64 GB

- Storage: 80gb (OS + Apps)

- OS: Oracle Linux Server release 5.11

With the topology as follows:

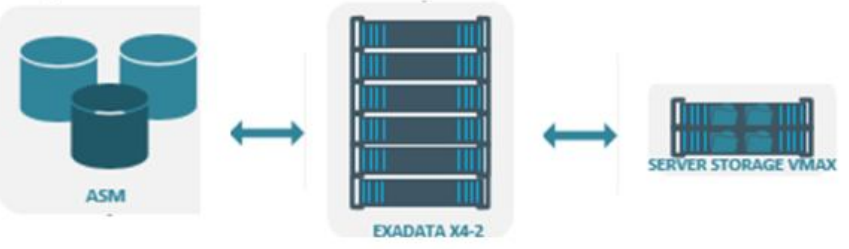

Figure 4: Backup Process Topology

For the Service Level Storage, there are levels to associate the disk speed with the desirable disk types stated on above. Here is the following table 8 for the type of configuration on VMAX:

Table 8: VMAX SLO performance

\begin{tabular}{|c|c|c|}
\hline SLO & $\begin{array}{c}\text { Minimum Required } \\
\text { Drive Combinations } \\
\text { to List SLO }\end{array}$ & Performance Expectation \\
\hline Diamond & SSD & Emulating SSD Performance \\
\hline Platinum & $\begin{array}{c}\text { SSD and }(15 \mathrm{~K} \text { or } \\
10 \mathrm{~K})\end{array}$ & $\begin{array}{c}\text { Emulating Performace between } 15 \mathrm{~K} \\
\text { Drive and SSD }\end{array}$ \\
\hline Gold & $\begin{array}{c}\text { SSD and }(15 \mathrm{~K} \text { or } 10 \mathrm{~K} \\
\text { or } 7.2 \mathrm{~K})\end{array}$ & Emulating 15K Drive Performance \\
\hline Silver & $\begin{array}{c}\text { SSD and }(15 \mathrm{~K} \text { or } 10 \mathrm{~K} \\
\text { or } 7.2 \mathrm{~K})\end{array}$ & Emulating 10K Drive Performance \\
\hline Bronze & $7.2 \mathrm{k}$ and $(15 \mathrm{k}$ or $10 \mathrm{k})$ & Emulating 7.2K Drive Performance \\
\hline Optimized & Any & System Optimized Performance \\
\hline
\end{tabular}


This experiment would only currently assess the platinum type which emulates the SAS level performance with $15 \mathrm{k}$ Drive above performance.

In this experiment, we will test 16 multiplex and compression level combination. Parameter combination with best scoring value will be the best parameter for this experiment.

Step by step for this experiment: prepare a RMAN backup folder and script containing the backup software configuration:

Prepare 2 folders:

a. backup_script: backup configuration script saving

b. backup_log: storing log files resulting from backup testing.

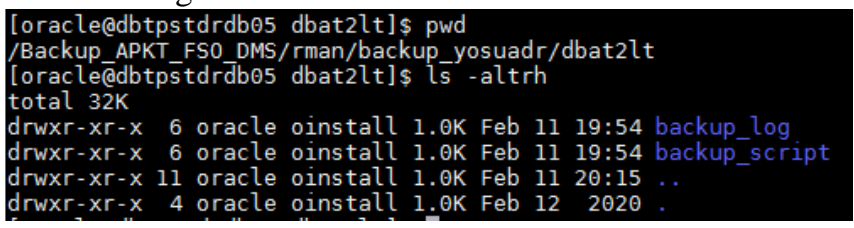

Figure 2: Backup Folder

Inside backup script folder, create 4 folders based on compression level.

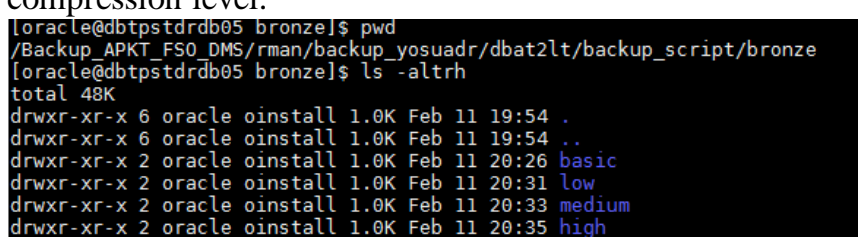

Figure 6: Bavkup Subfolder (Compression Level)

Within each folder the compression level will create a backup script based on the multiplexing level to be tested:

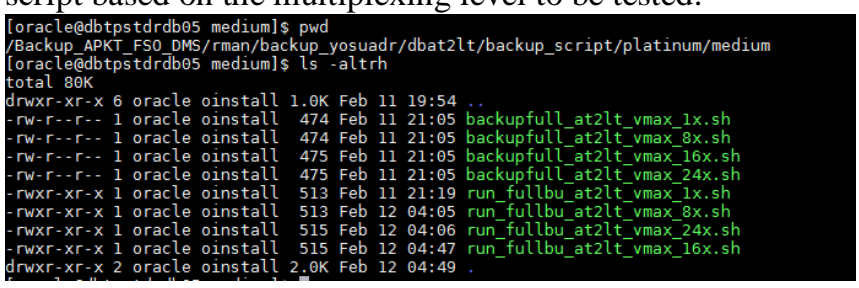

Figure 7: Script Backup (Multiplexing Level)

The output results are compared to measure output size backup, time taken and output rate to find out the most optimal backup software and infrastructure for each database being tested.

Data collection test results using sqlplus software (figure 8) as follows:

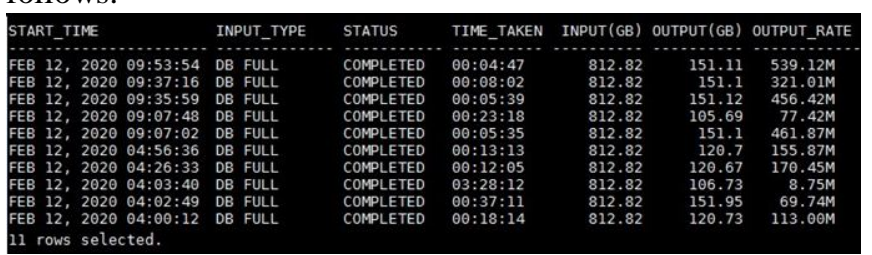

Figure 8 :Test Result by Sqlplus
Then from the log file that has been created will be matched with the recap:

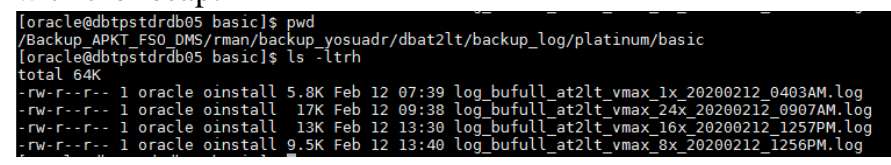

Figure 10: Log File Backup Process

We set a scenario with the parameter discussed on the above. We look into 4 types of different compression types with compression divided into 4 multiplexing scenarios $(1 \mathrm{x}, 8 \mathrm{x}$, $16 x$ and 24x) on a Platinum Service Level Storage. The result is as follow (table 9):

Table 9:Experiment Result

\begin{tabular}{|c|c|c|c|c|c|}
\hline Compression & $\begin{array}{c}\text { Multiple } \\
\text { xing }\end{array}$ & $\begin{array}{c}\text { INPUT } \\
\text { SIZE } \\
(\mathbf{G B})\end{array}$ & $\begin{array}{c}\text { Output } \\
\text { Size (GB) }\end{array}$ & Time Taken & $\begin{array}{c}\text { CPU } \\
\text { Usage } \\
(\%)\end{array}$ \\
\hline \multirow{4}{*}{ BASIC } & $1 \mathrm{x}$ & 812,82 & 106,73 & $03: 28: 12$ & 11,94 \\
\cline { 2 - 6 } & $8 \mathrm{x}$ & 812,82 & 106,10 & $00: 35: 42$ & 23,42 \\
\cline { 2 - 6 } & $16 \mathrm{x}$ & 812,82 & 106,01 & $00: 25: 10$ & 31,57 \\
\cline { 2 - 6 } & $24 \mathrm{x}$ & 812,82 & 105,69 & $00: 23: 18$ & 41,91 \\
\hline \multirow{5}{*}{ LOW } & $1 \mathrm{x}$ & 812,82 & 151,95 & $00: 37: 11$ & 11,59 \\
\cline { 2 - 6 } & $8 \mathrm{x}$ & 812,82 & 151,10 & $00: 08: 02$ & 18,30 \\
\cline { 2 - 6 } & $16 \mathrm{x}$ & 812,82 & 151,10 & $00: 05: 35$ & 15,98 \\
\cline { 2 - 6 } & $24 \mathrm{x}$ & 812,82 & 151,11 & $00: 04: 47$ & 41,91 \\
\hline \multirow{5}{*}{ MEDIUM } & $1 \mathrm{x}$ & 812,82 & 120,56 & $01: 46: 25$ & 11,94 \\
\cline { 2 - 6 } & $8 \mathrm{x}$ & 812,82 & 120,73 & $00: 18: 14$ & 18,55 \\
\cline { 2 - 6 } & $16 \mathrm{x}$ & 812,82 & 120,70 & $00: 13: 13$ & 28,68 \\
\cline { 2 - 6 } & $24 \mathrm{x}$ & 812,82 & 120,67 & $00: 12: 05$ & 29,59 \\
\hline \multirow{5}{*}{ HIGH } & $1 \mathrm{x}$ & 812,82 & 79,26 & $15: 55: 25$ & 23,42 \\
\cline { 2 - 6 } & $8 \mathrm{x}$ & 812,82 & 79,54 & $03: 46: 35$ & 34,99 \\
\cline { 2 - 6 } & $16 \mathrm{x}$ & 812,82 & 78,87 & $02: 44: 25$ & 32,92 \\
\cline { 2 - 6 } & $24 \mathrm{x}$ & 812,82 & 78,66 & $01: 33: 09$ & 46,75 \\
\hline
\end{tabular}

We then convert the result into scores based on the threshold defined:

Table 10: Experiment Scoring

\begin{tabular}{|c|c|c|c|c|c|c|}
\hline Compression & $\begin{array}{c}\text { Multiple } \\
\text { xing }\end{array}$ & $\begin{array}{c}\text { Output } \\
\text { Size } \\
\text { (Score) }\end{array}$ & $\begin{array}{c}\text { Time } \\
\text { Elapsed } \\
\text { (Score) }\end{array}$ & $\begin{array}{c}\text { Output } \\
\text { Rate } \\
\text { (Score) }\end{array}$ & $\begin{array}{c}\text { CPU } \\
\text { Usage } \\
\text { (Score) }\end{array}$ & Total \\
\hline \multirow{4}{*}{ BASIC } & $1 \mathrm{x}$ & 7 & 3 & 3 & 9,5 & 22,5 \\
\cline { 2 - 7 } & $8 \mathrm{x}$ & 7 & 6,5 & 5 & 7 & 25,5 \\
\cline { 2 - 7 } & $16 \mathrm{x}$ & 7 & 7 & 5 & 5 & 24 \\
\cline { 2 - 7 } & $24 \mathrm{x}$ & 7 & 7 & 5 & 3 & 22 \\
\hline \multirow{4}{*}{ LOW } & $1 \mathrm{x}$ & 3 & 6,5 & 5 & 10 & 24,5 \\
\cline { 2 - 7 } & $8 \mathrm{x}$ & 3 & 9 & 9 & 8 & 29 \\
\cline { 2 - 7 } & $\underline{16 \mathrm{x}}$ & $\underline{\mathbf{3}}$ & $\underline{\mathbf{1 0}}$ & $\underline{\mathbf{1 0}}$ & $\mathbf{9}$ & $\underline{\mathbf{3 2}}$ \\
\cline { 2 - 7 } & $24 \mathrm{x}$ & 3 & 10 & 10 & 3 & 26 \\
\hline \multirow{4}{*}{ MEDIUM } & $1 \mathrm{x}$ & 6 & 5 & 4 & 10 & 25 \\
\cline { 2 - 7 } & $8 \mathrm{x}$ & 6 & 7 & 6 & 8 & 27 \\
\cline { 2 - 7 } & $16 \mathrm{x}$ & 6 & 8 & 8 & 6 & 28 \\
\cline { 2 - 7 } & $24 \mathrm{x}$ & 6 & 8 & 8 & 6 & 28 \\
\hline \multirow{4}{*}{ HIGH } & $1 \mathrm{x}$ & 10 & 1 & 2 & 7 & 20 \\
\cline { 2 - 7 } & $8 \mathrm{x}$ & 10 & 3 & 3 & 5 & 21 \\
\cline { 2 - 7 } & $16 \mathrm{x}$ & 10 & 4 & 3 & 5 & 22 \\
\cline { 2 - 7 } & $24 \mathrm{x}$ & 10 & 5 & 4 & 3 & 22 \\
\hline
\end{tabular}

From this study case we can take the conclusion that the most suggested parameter tuning for this study case of a Hot Backup Process with 812,12 GB size file is better with Low Compression and 16x Multiplexing on a Platinum Service Level Storage, for it only takes the time of 05:35 Minutes for compressing a $812,82 \mathrm{~GB}$ file into $151,11 \mathrm{~GB}$ and only taking $15,98 \%$ of CPU Usage. 
Other noteworthy result is that the High Compression based can compress up to 10:1 size ratio on the cost of using at least $23.5 \%$ of the resource with a very long runtime or $46,75 \%$ of CPU resource with at least 1.5 hours runtime.

Basic and medium compression are also head to head in scoring with only a slight difference for $8 \mathrm{x}$ multiplexing rate and above, if the user is going for more efficient space storage, it is suggested to use the medium compression for the slight difference in time

\section{CONCLUSION}

Based on experiment and the result, This study success to show experiment level on how does the performance of each method using Oracle RMAN. The Experiment Result proven how each parameter tuning would affect the backup performance on Oracle database, since it is only categorized on high-level language (basic, high, low, medium) and the level of multiplexing or parallelism process.

For further works, experiments can use a variety of database sizes, from small database to large database so that it can be seen which parameters are more effectively for small database and large database.

\section{REFERENCES}

[1] T. M. Connolly and C. E. Begg, "A Constructivist-Based Approach to Teaching Database Analysis and Design," Journal of Information Systems Education, Vol. 17(1), 2006.

[2] R. Schiesser, "Prentice Hall," in IT Systems Management (2nd ed.), 2010.

[3] Y. Son, J. Choi, J. Jeon, C. Min, S. Kim, H. Y. Yeom and H. Han, "SSD-assisted Backup and Recovery for Database Systems," IEEE 33rd International Conference on Data Engineering, pp. 285-296, 2017. https://doi.org/10.1109/ICDE.2017.88

[4] Oracle, "Tuning Oracle Recovery Manager," Oracle White Paper, 2001.

[5] R. Cegiela, "Selecting Technology for Disaster Recovery," in International Conference on Dependability of Computer Systems, 2006.

[6] K. Sharma and K. R. Singh, "Online data back-up and disaster recovery techniques in cloud computing: A review.," International Journal of Engineering and Innovative Technology (IJEIT), vol. 2, no. 5, pp. 249-254, 2012.

[7] Q. Li and H. Xu, "Research on the Backup Bechanism of Oracle Database," International Conference on Environmental Science and Information Application Technology. Vol. 2., pp. 423-426, 2009. https://doi.org/10.1109/ESIAT.2009.294
[8] X. J. Liu, "A brief analysis of the disaster recovery backup technology in oracle database dataguard.," 2nd International Conference on Industrial and Information Systems, vol. 2, pp. 234-236, 2010.

[9] Oracle, "Oracle Advanced Compression," Oracle White Paper, 2019.

[10] TheGeekDiary. [Online]. Available: https://www.thegeekdiary.com/beginners-guide-to-rma n-compression-for-backups/.

[11] "SourceWare," [Online]. Available: https://www.sourceware.org/bzip2/.

[12] "lzop," [Online]. Available: https://www.lzop.org/.

[13] "zlib Applications," [Online]. Available: http://zlib.net/apps.html.

[14] WysheidTeam, "General DBA Activities," [Online]. Available: https://www.wysheid.com/blog/general-dba-activities/r man-compressed-backup-learn-reduce-size-oracle-datab ase-backups/.

[15] M. Hoseinzadeh, "A Survey on Tiering and Caching in High-Performance Storage Systems," arXiv preprint, 2019.

[16] Aventi. [Online]. Available: (https://www.aventissystems.com/blog-Hard-Drive-Co mparison-SATA-SAS-SSDs-s/12079.htm) .

[17] "docs.oracle," [Online]. Available: https://docs.oracle.com/cd/B28359_01/server.111/b283 10/archredo002.htm\#ADMIN11330. 